\title{
BMJ
}

\section{Effectiveness of acute geriatric units on functional decline, living at home, and case fatality among older patients admitted to hospital for acute medical disorders: meta- analysis}

\begin{abstract}
Juan J Baztán, consultant geriatrician, ${ }^{1}$ Francisco M Suárez-García, geriatrician, ${ }^{2}$ Jesús López-Arrieta, consultant geriatrician, ${ }^{3}$ Leocadio Rodríguez-Mañas, chief of department, ${ }^{4}$ Fernando Rodríguez-Artalejo, professor of preventive medicine and public health ${ }^{5,6}$
\end{abstract}

\begin{abstract}
Objective To assess the effectiveness of acute geriatric units compared with conventional care units in adults aged 65 or more admitted to hospital for acute medical disorders.
\end{abstract}

Design Systematic review and meta-analysis.

Data sources Medline, Embase, and the Cochrane Library up to 31 August 2008, and references from published literature.

Review methods Randomised trials, non-randomised trials, and case-control studies were included. Exclusions were studies based on administrative databases, those that assessed care for a single disorder, those that evaluated acute and subacute care units, and those in which patients were admitted to the acute geriatric unit after three or more days of being admitted to hospital. Two investigators independently selected the studies and extracted the data.

Results 11 studies were included of which five were randomised trials, four non-randomised trials, and two case-control studies. The randomised trials showed that compared with older people admitted to conventional care units those admitted to acute geriatric units had a lower risk of functional decline at discharge (combined odds ratio $0.82,95 \%$ confidence interval 0.68 to 0.99 ) and were more likely to live at home after discharge $(1.30,1.11$ to 1.52$)$, with no differences in case fatality $(0.83,0.60$ to 1.14). The global analysis of all studies, including nonrandomised trials, showed similar results.

Conclusions Care of people aged 65 or more with acute medical disorders in acute geriatric units produces a functional benefit compared with conventional hospital care, and increases the likelihood of living at home after discharge.

\section{INTRODUCTION}

Adequate hospital care for older people ( $\geq 65$ years) with acute medical disorders requires a comprehensive assessment by multidisciplinary teams to detect early those patients at highest risk of functional decline and institutionalisation. ${ }^{1-3}$ Such care also requires early planning for discharge, and follow-up. ${ }^{1-3}$ The primary aim of this model of care is to reduce functional decline, which is the main determinant of quality of life, cost of care, and vital prognosis. ${ }^{4}$ Delaying functional decline and increasing the chances of living at home are at least as important as reducing case fatality in frail older people. ${ }^{2}$

Several interventions have been proposed to improve the effectiveness and efficiency of hospital care for older people with acute medical disorders. Assessment of geriatric patients by a multidisciplinary consultation team has not, however, shown benefits for case fatality, functional decline, or place of residence at discharge. ${ }^{25}$ Hospital at home care for elderly medical patients reduces hospital stay but increases overall length of care, and objective evidence of economic benefit is insufficient. ${ }^{6}$ Another type of intervention is that provided by multidisciplinary geriatric teams in "acute care for elders units" or acute geriatric units. Previous reviews lumped such units with geriatric evaluation and management units, which normally care for elderly people after stabilisation of an acute condition. $^{27-9}$ Other reviews have focused on partial aspects of acute care such as discharge planning or physiotherapy. ${ }^{1011}$ To our knowledge only one review has been published on the effectiveness of acute geriatric units, and that provided only descriptive data. $^{12}$

We systematically reviewed studies on the effect of acute geriatric units compared with conventional hospital care in the treatment of older people with acute medical disorders. We hypothesised that acute geriatric units would reduce functional decline, increase the proportion of patients able to live at home after discharge, reduce case fatality, and result in shorter hospital stays than conventional hospital units.

\section{METHODS}

Our review included randomised trials, non-randomised trials, and case-control studies that compared 
Articles identified by search of title or abstract $(n=4537)$

$\downarrow$

Retrieved for more detailed evaluation $(n=124)$ :

Published $(\mathrm{n}=122)$

Unpublished $(n=2)$

Rejected $(n=112)$ :

Consulting team $(n=23)^{\text {w13-w35 }}$

Medical stability-subacute care $(n=17)^{\text {w36-w52 }}$

Acute and subacute care $(n=6)^{\text {w5 }}{ }^{\text {-w5 }} 8$

Age $<65$ years $(n=4)^{\text {w59-w62 }}$

Hospital administrative database $(n=6)^{\text {w63-w68 }}$

$\rightarrow$ Historical cohorts $(n=1)^{\text {w69 }}$

Non-comprehensive multidisciplinary intervention $(n=7)^{\text {w70-w76 }}$

Intervention not described $(\mathrm{n}=2)^{\mathrm{w} 77}$ w78

Descriptive studies $(n=15)^{\text {w79-w93 }}$

Non-hospital interventions $(\mathrm{n}=5)^{\text {w94-w98 }}$

Other $(n=12)^{\text {w99-w110 }}$

Reviews $(n=14)$

$\checkmark$

Included in review $(\mathrm{n}=11)$ :

Randomised controlled studies $(n=5)^{\text {w1-w6 }}$

Controlled studies $(n=6)^{\text {w7-w12 }}$

Fig 1| Flow of papers through study

outcome of care in acute geriatric units with that in conventional hospital units in patients aged 65 years or more with acute medical disorders. Acute geriatric units were defined as hospital units with their own physical location and structure and run by a specialised multidisciplinary team with direct responsibility for the care of elderly people with acute medical disorders, including acute exacerbations of chronic diseases. This definition is based on seminal research on acute geriatric units ${ }^{12 \mathrm{w} 36}$ and is consistent with previous definitions in this discipline; for example, two studies stated that an acute geriatric unit is an interdisciplinary model for providing care to older adults during admission to hospital for an acute medical illness. ${ }^{413}$ These studies agreed with those of other authors that the acute geriatric unit is a defined medical unit that uses an interdisciplinary team specialising in geriatric care to provide increased attention to a patient's level of functioning, specific treatment of diagnoses common to older people, and integrated planning of discharge to maximise clinical outcomes. ${ }^{413 \mathrm{w} 5 \mathrm{w} 9 \mathrm{w} 10}$ Patients are admitted to acute geriatric units for acute problems such as pneumonia, heart failure, sepsis, delirium, urinary tract infections, or chronic obstructive pulmonary disease, which do not require treatment in other specialised units (for example, intensive care unit or coronary unit).

We excluded studies that used secondary data from databases designed for the purposes of healthcare administration, with information not collected directly from the patients by the researchers. We also excluded studies that evaluated interventions aimed at a specific medical disorder (for example, stroke units, psychogeriatric units) or surgical process (for example, orthogeriatric units). To ensure we did not include studies that evaluated care provided out of the acute phase we excluded studies that assessed units with care lasting beyond the acute phase and those with patients admitted to the intervention unit three or more days after hospital admission.

\section{Outcome variables}

The primary outcomes were functional decline, living at home, and case fatality at discharge and three months later. We considered functional decline to be loss of independence in one or more basic activities of daily living compared with the situation before admission. ${ }^{414}$ To assess basic activities of daily living we used the Katz index and the Barthel index; each basic activity (transfers, mobility, dressing, eating, bathing) was classified as independent or dependent according to the need for physical assistance to carry out the activity. ${ }^{14 \mathrm{w} 3 \mathrm{w} 6 \mathrm{w} 10}$ The secondary outcomes were hospital stay during the index admission, the cost of the index admission, and readmission at three months after discharge.

\section{Literature search and data extraction}

We searched Medline, Embase, and the Cochrane central register of controlled trials up to 31 August 2008 (see web extra appendix for search strategy) and scanned the reference lists of reviews and relevant articles. We included articles in English and Spanish. To obtain information on unpublished studies or to complete data for published studies on the hospital unit, patients' characteristics, hospital stay, and costs we contacted the authors.

Two investigators (JJB and FS) evaluated the eligibility and methodological quality of studies obtained from the literature search. In cases of discrepancy a third researcher (JLA) reviewed the studies, and agreement was reached by consensus. JJB and FS independently extracted and compared the data.

\section{Quality assessment}

For randomised trials we extracted information on concealment of allocation, the proportion of patients lost to follow-up, the use of intention to treat analysis, and whether the assessment of outcomes was done blinded to intervention group. For non-randomised trials we recorded only losses to follow-up and type of outcome assessment.

We summarised the overall quality of randomised trials with the Jadad scale, excluding blinding of the intervention as this is not possible for acute geriatric units. ${ }^{915}$ Thus the scores ranged from 0 to 3. For all studies we also used the Van Tulder scale, scored from 0 to 19 (highest quality), which has been used in other systematic reviews of interventions in the care of elderly people. ${ }^{16}$

\section{Data synthesis}

For dichotomous outcomes we give the results as combined odds ratios with $95 \%$ confidence intervals and for quantitative outcomes as differences in means with standard errors. We used fixed effects methods to combine the outcomes across studies, except when 


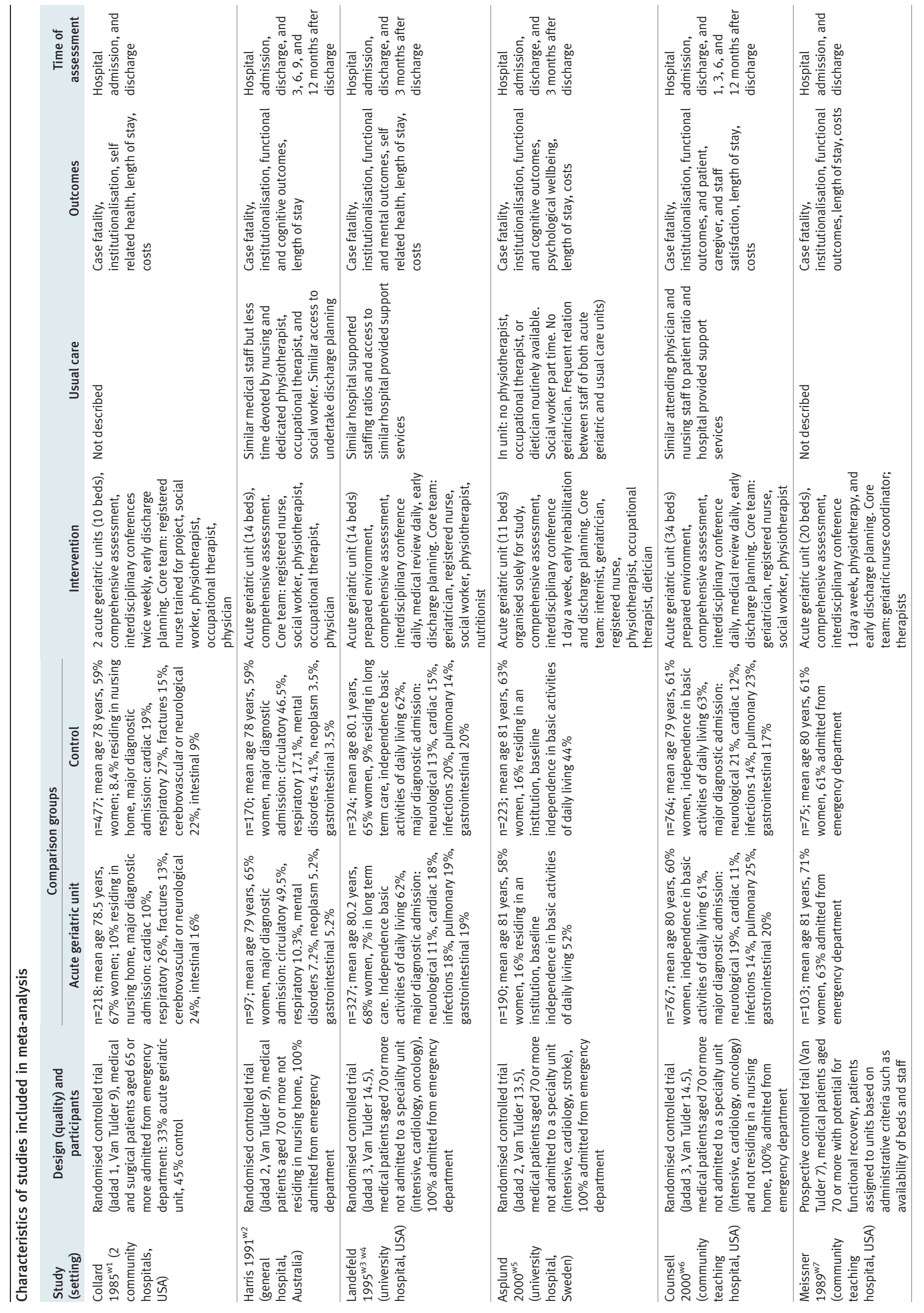

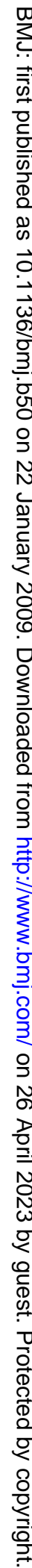




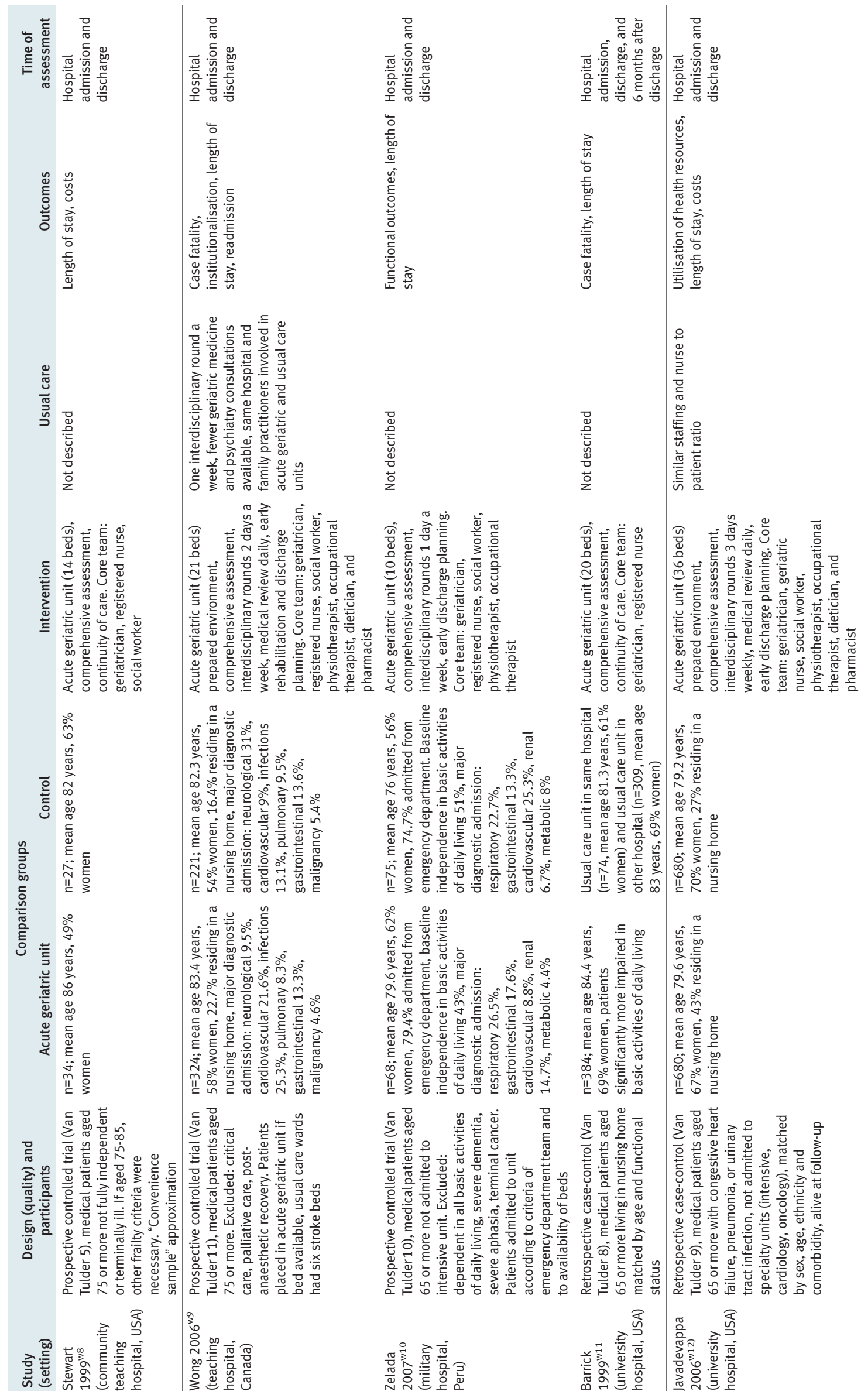

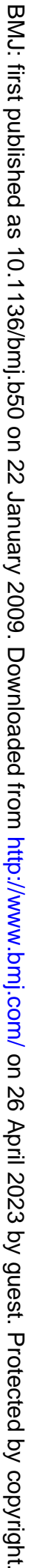


important heterogeneity was observed. Heterogeneity was quantified with the $\mathrm{I}^{2}$ statistic, which measures the percentage of variation among studies due to heterogeneity rather than to chance. For practical purposes we considered heterogeneity to be important when $\mathrm{I}^{2}$ was more than $30 \%$. When it was considered appropriate to combine results in these cases, we used random effects methods. ${ }^{17}$

When we were unable to retrieve the standard deviation for length of hospital stay and cost of hospital admission, we approximated it from the standard error and $95 \%$ confidence intervals. ${ }^{18}$ When we were unable to estimate the standard deviation, we contacted the authors; this was provided for one study. ${ }^{\text {w6 }}$ In three studies $^{\mathrm{w} 1 \mathrm{w} 3 \mathrm{w} 11}$ we had to assume that the standard deviation was the same as the mean. Finally, given that we had specific data from each of the two centres that participated in one of the studies, ${ }^{\text {w1 }}$ we considered them as independent studies for the analysis of hospital stay and its cost.

Although we created funnel plots to identify possible publication biases, these proved difficult to interpret given the small number of studies. Statistical analyses were carried out using RevMan 4.3 (Cochrane Collaboration).

\section{RESULTS}

The literature search yielded 4038 articles. After review of the titles and abstracts 119 articles were selected for critical reading. Twelve articles totalling 11 studies met the inclusion criteria (fig 1). Seven of these were done in the United States and the remainder in Australia, Canada, Sweden, and Peru. Authors of five studies were contacted for additional data, ${ }^{\mathrm{w} 3} \mathrm{w} 5 \mathrm{w} 6 \mathrm{w}$ 9 $\mathrm{w10}$ but for only one ${ }^{\mathrm{w} 6}$ was unpublished data on patients' living at home after discharge and at three months used.

The table presents the principal characteristics of the included studies. Five were randomised trials, ${ }^{\text {w1-w6 }}$ four were non-randomised trials, ${ }^{\mathrm{w} 7 \mathrm{w} 10}$ and two were retrospective case-control studies. ${ }^{\text {w11 w12 }}$

The methodological quality of the studies was heterogeneous, especially for non-randomised trials (table). Three of the randomised trials used sealed envelopes to allocate patients to groups. ${ }^{\text {w3 w5 w6 }}$ Only one of them did an intention to treat analysis at discharge, but this was not done at follow-up. ${ }^{\text {w6 }}$ Of the

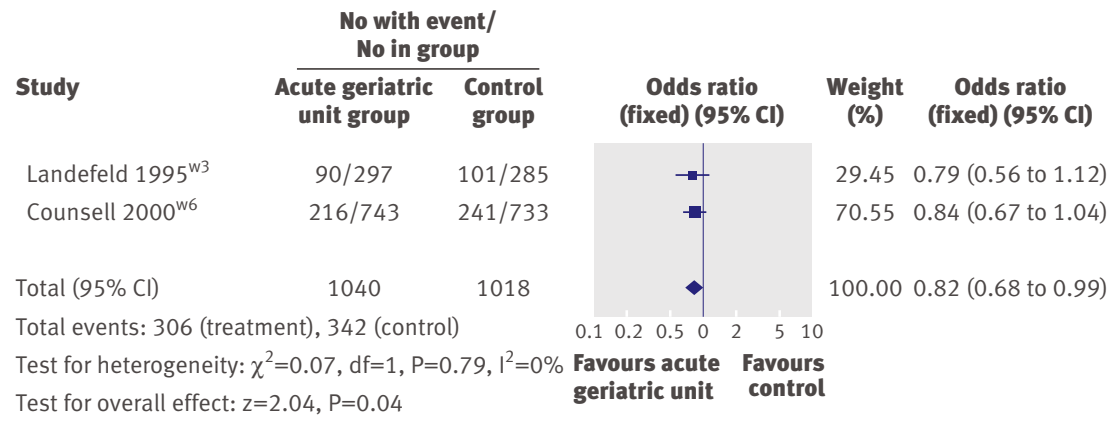

Fig 2 Functional decline at discharge from hospital in randomised trials comparing acute geriatric units with conventional hospital care four non-randomised trials, three used informal procedures for allocation based on bed availability. ${ }^{\mathrm{w} 7}$

Criteria for patient selection were age plus a medical condition not requiring admission to specialty units. Five studies included patients aged 70 or more, ${ }^{\text {w2 w7 }}$ three aged 65 or more, ${ }^{\text {w1 w1 } 10}$ w11 and one aged 75 or more. ${ }^{\text {w9 }}$ One study selected patients aged 75 or more with at least one geriatric condition. ${ }^{\mathrm{w} 8}$ Finally, one study selected patients aged 65 or more who were admitted for heart failure, pneumonia, or urinary tract infection. $^{\text {w12 }}$ Two studies excluded older people ( $\geq 70$ years) living in a nursing home, ${ }^{\text {w2 w6 }}$ whereas another ${ }^{\text {w11 }}$ included only those who lived in a nursing home.

In four of the five randomised trials all the patients came from emergency services, ${ }^{\text {w2-w6 }}$ whereas in three non-randomised studies more than $62 \%$ came from emergency services. ${ }^{\text {w7 } 9 \text { w10 }}$ Three studies did not provide this information. ${ }^{\mathrm{w} 8 \mathrm{w} 11 \mathrm{w} 12}$

The intervention units functioned in similar ways, generally having four characteristics that distinguished them from conventional units: comprehensive geriatric assessment of patients, use of standardised instruments for measurements, weekly multidisciplinary meetings, and early planning of discharge. The composition of the basic multidisciplinary team typically included at least one geriatrician, nursing staff trained in geriatrics, a social worker, and therapists.

The follow-up period varied. All the randomised studies except one ${ }^{\text {w1 }}$ provided follow-up data at three months, at a minimum. Five studies reported on readmissions, two at three months after discharge $\mathrm{w}^{\mathrm{w} 3 \mathrm{w5}}$ and three at one month after discharge. ${ }^{\mathrm{w} 6 \mathrm{w} 9 \mathrm{w} 11}$

\section{Functional decline}

Only three studies presented results on functional decline at discharge, two of which were randomised trials (fig 2). In these two studies, which accounted for more than $65 \%$ of patients in the randomised trials, the patients in acute geriatric units showed a lower risk of functional decline than those in conventional units (combined odds ratio $0.82,95 \%$ confidence interval 0.68 to 0.99 ). After the inclusion of a non-randomised study, results were similar $(0.78,0.65$ to 0.94$)$ but showed greater heterogeneity $\left(\mathrm{I}^{2}=55.7 \%\right)$ because of the large reduction in functional decline in the acute geriatric unit in that study $(0.35,0.17$ to 0.76$){ }^{\text {w10 }}$

Only one study ${ }^{\mathrm{w} 6}$ provided data on functional decline at three months after discharge, with no differences in the incidence of functional decline between the groups. Another three randomised studies provided data that could not be analysed but reported an absence of differences in functional decline at three months after discharge. ${ }^{\text {w2 w3 w5 }}$

\section{Living at home}

In randomised studies, patients cared for in acute geriatric units were more likely to be living at home after discharge (1.30, 1.11 to 1.52$)$, a benefit that was 


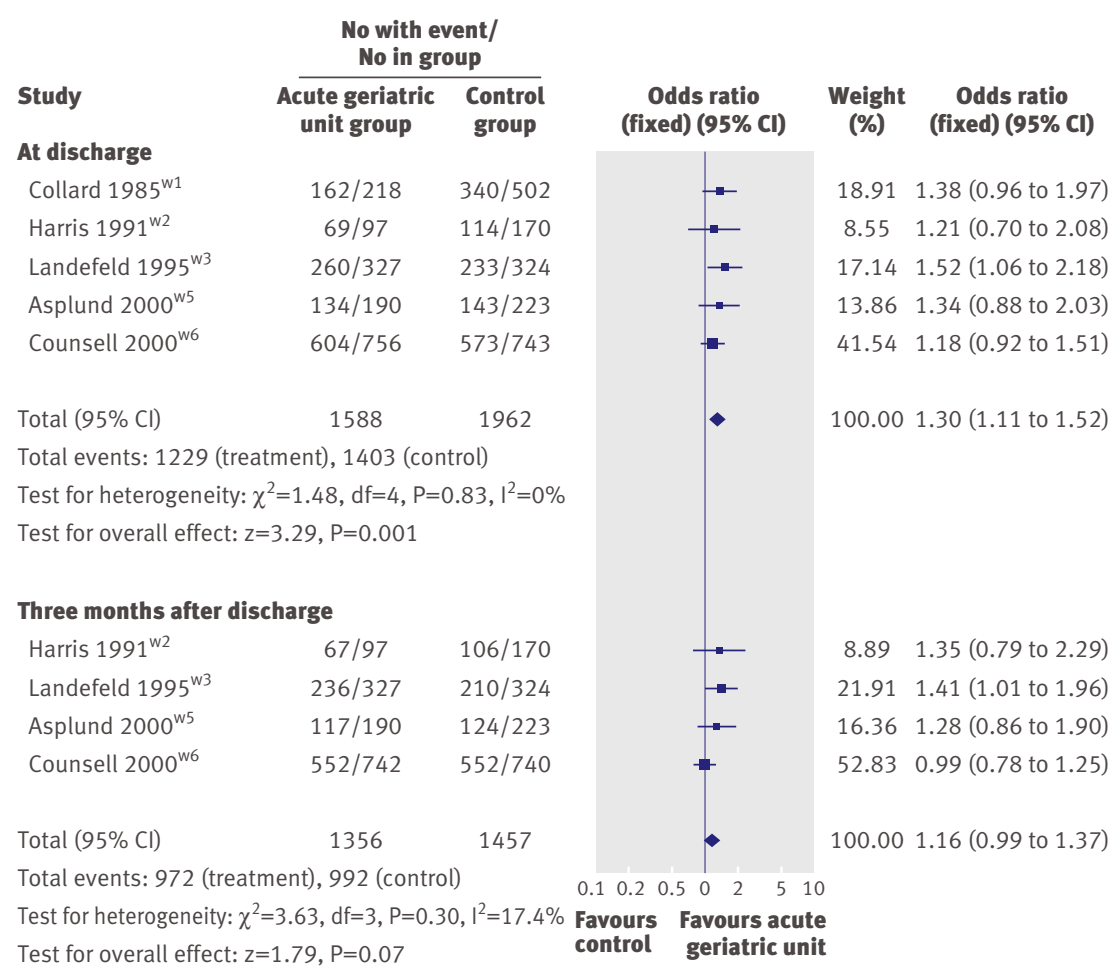

Fig 3 | Living at home at discharge from hospital and three months after discharge in randomised trials comparing acute geriatric units with conventional hospital care

marginally maintained three months after discharge (fig 3). Results at discharge still held when analyses were repeated including non-randomised studies $(1.28$, 1.12 to 1.47$)$.

Acute geriatric units and conventional care units showed no differences in frequency of admission to a nursing home at discharge $(0.76,0.51$ to 1.28$)$ or three months after discharge $(0.90,0.74$ to 1.14$)$.

\section{Case fatality}

No significant differences were found in case fatality between acute geriatric units and conventional care units either in hospital or three months after discharge (fig 4). The results were similar in the randomised and non-randomised studies, although in the randomised studies case fatality at discharge ranged between $2.5 \%$ and $9 \%$ whereas in the non-randomised studies it exceeded $10 \%$.

\section{Length of hospital stay and cost of admission}

All the included studies reported on length of hospital stay, but three did not report dispersion measures. ${ }^{\text {w1 w3 w11 }}$ The length of stay in the acute geriatric units was fewer than 12 days, and fewer than nine days in the studies published from 1995. In nine of the 11 studies a trend was observed towards a reduced length of stay, of

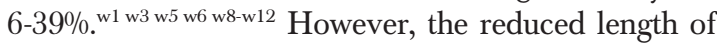
stay was heterogeneous among studies; $\mathrm{I}^{2}$ was $49.4 \%$ in the randomised studies and $74.1 \%$ in the non-randomised studies. This heterogeneity limits firm conclusions being reached on this outcome.
Data on the cost of hospital stay were reported in four of the five randomised studies ${ }^{\mathrm{w} 1 \mathrm{w} 3-\mathrm{w} 6}$ and in three of the six non-randomised studies. ${ }^{\text {w7 w8 w12 }}$ The US dollar was used in all studies except for one that used Swedish crowns, with the dollar conversion calculated according to the exchange rate provided by the authors. ${ }^{\mathrm{w} 5}$ Although the data are difficult to interpret because they did not allow cost effectiveness ratios to be estimated, a slightly, yet significant, lower cost of hospital care was found in acute geriatric units (combined mean difference $-0.31,95 \%$ confidence interval -0.52 to -0.09 ; $\left.\mathrm{I}^{2}=0 \%\right)$.

Two randomised studies ${ }^{\mathrm{w} 3} \mathrm{w} 5$ provided data on readmissions to hospital at three months after discharge, and another randomised study ${ }^{\mathrm{w} 6}$ and two nonrandomised studies reported on readmissions in the month after discharge. ${ }^{\text {w9 w11 }}$ Another two studies ${ }^{\text {w7 w12 }}$ provided data in a format that was not useful for analysis. The combined odds ratio for readmission at three months in patients discharged from acute geriatric units compared with conventional units in all studies was 0.93 (95\% confidence interval 0.77 to 1.11) and in the randomised studies was 1.11 (0.92 to $1.35)$.

\section{Sensitivity analysis}

Separate analyses for the randomised studies and the other studies provided similar results, except that the reduction in hospital stay was larger in the nonrandomised studies. The analysis was repeated after excluding the oldest studies (those published before 1995 and that lacked a geriatrician), and after assuming adverse results in people lost to follow-up or lacking data on outcomes. No substantial changes in the results were found in any of these cases.

\section{DISCUSSION}

The results of this meta-analysis suggest that care of older people ( $\geq 65$ years) with acute medical disorders in acute geriatric units leads to less functional decline at discharge and a higher probability of living at home after discharge. The effect of geriatric assessment has been evaluated in different care settings. ${ }^{8}$ In the case of patients admitted to hospital for acute medical disorders, only the activity of multidisciplinary consultation teams has been assessed, with no benefits found for case fatality, functional decline, or living at home after discharge. ${ }^{2}$ Since admission to hospital is a risk factor for case fatality, functional decline, and admission to a nursing home, any intervention that helps reduce this risk is potentially important. ${ }^{19}$

The $18 \%$ reduction in functional decline associated with acute geriatric units is similar to that found in a study of patients aged 65 or more with acute medical disorders who received physiotherapy within multidisciplinary care.${ }^{11}$ Furthermore, the benefit of living at home after discharge was comparable to that reported in another study in the combined analysis of acute and subacute hospital care units. ${ }^{2}$

This improvement in functional outcomes was not accompanied by an increased rate of admissions at 
three months or increased case fatality. Although the tendency towards a higher probability of living at home was maintained at three months after discharge from an acute geriatric unit, the reduction in functional decline was seen only at discharge; thus future studies should examine whether it persists in the medium term. We did not find a reduction in case fatality either at discharge or at three months follow-up. This is a common finding in studies of very old (frequently aged 80 or more) or frail people. In fact, several authors and older people too feel that improving case fatality at the expense of disability and dependence should not be considered an optimal outcome. ${ }^{220}$

\section{Characteristics of acute geriatric unit}

The studies reviewed provide limited information about the characteristics and form of operation of the conventional hospital units. This is important because the effect of acute geriatric units is measured by comparison with these units and could vary locally depending on the characteristics of conventional hospital units. Although the acute geriatric units in this review all included therapists as part of the normal working team (table), in general they did not have more staff than the conventional units, so that the differences between them seem to centre on specialisation and organisation of work. The distinctive feature of acute geriatric units is the comprehensive geriatric assessment and care focusing on patients' needs, interdisciplinary work carried out by a core team of professionals (geriatrician, nursing staff trained in geriatrics, therapists, and social worker), and early

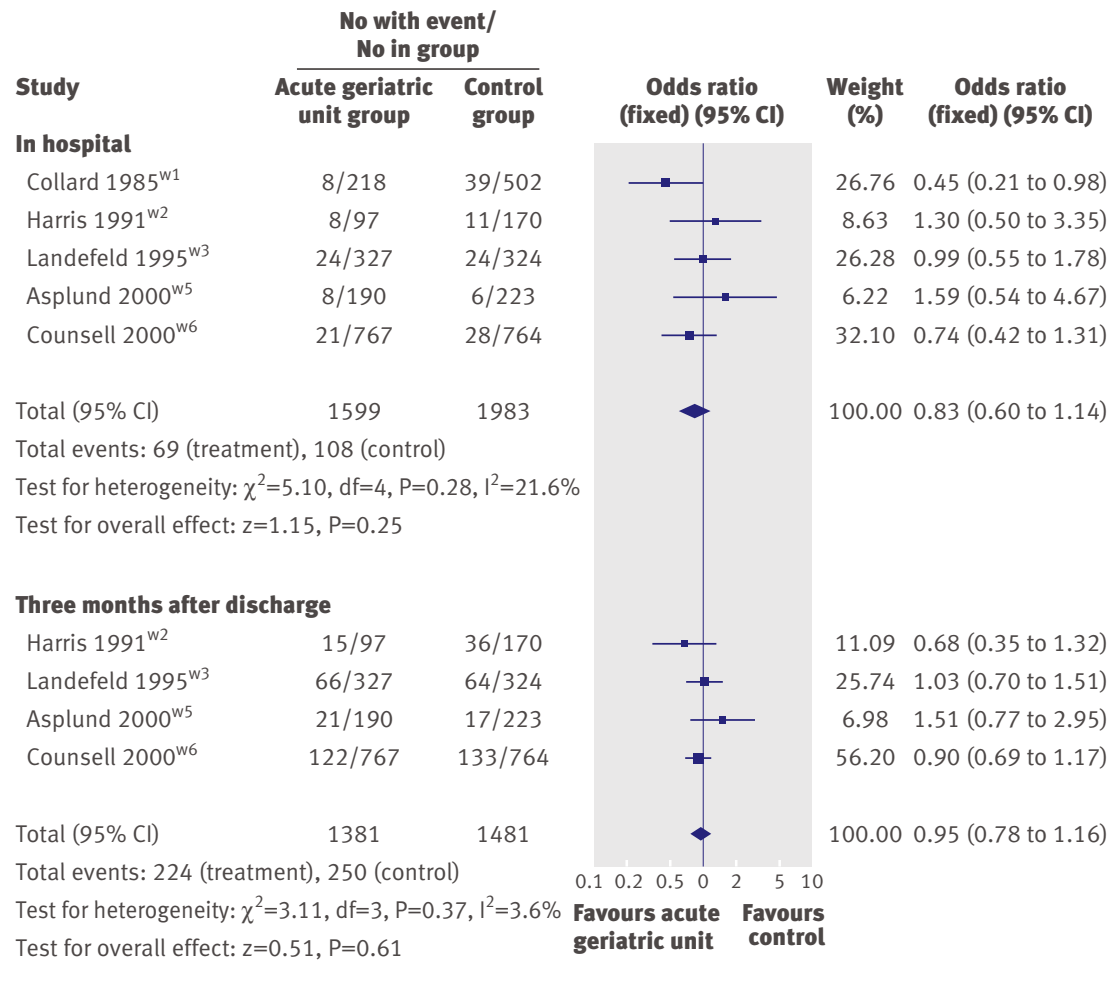

Fig 4 Case fatality in hospital and three months after discharge in randomised trials comparing acute geriatric units with conventional hospital care planning of discharge. Other studies have evaluated partial aspects of this specialised care, such as early discharge planning or physiotherapy alone, ${ }^{1011}$ without finding conclusive results, indicating that the benefit may derive from a combination of these inter ventions. ${ }^{2}$ Specialisation in the care of elderly people and formal interdisciplinary meetings (ranging from once a week in three studies to more than once a week in five studies) may contribute to the benefits of care in acute geriatric units, as has been shown in patients with stroke or hip fracture. ${ }^{2122}$ Another aspect of acute geriatric units that might contribute to their effectiveness, compared with the lack of benefits from geriatric consultation teams, is direct responsibility for the patient, which ensures compliance with diagnostic and therapeutic recommendations and the implementation of the care plan. ${ }^{2 \mathrm{w} 29}$

In general the working method of the acute geriatric unit is similar to that of the geriatric evaluation and management unit, although more intensive. These two units complement each other in providing care; in some places, such as the United Kingdom, services may even be found where care in the acute hospital phase is complemented by subsequent care in the same unit to promote functional recovery and return home. ${ }^{23}$ The benefit of care in the subacute phase is better established, however, since geriatric evaluation and management units are the units most often studied in evaluating the impact of comprehensive geriatric assessment in hospital. ${ }^{28}$ Separate analysis of intervention in the acute hospital phase makes it possible to assess the impact of early specialised intervention in older people. The incidence of functional decline is highest in this early phase, ${ }^{1424}$ which increases the risk of admission to a nursing home and death. ${ }^{25}$

\section{Patients and case mix}

The operational definition of acute geriatric unit used in this review was inclusive, allowing information to be combined across healthcare systems in different parts of the world. This serves to increase generalisability of results while retaining internal validity, because metaanalytic techniques ensure that the data for each acute geriatric unit are compared only with those of the standard care group in each trial. A potential drawback of combining data across healthcare systems is that our results might not apply to those systems where standards of care had evolved substantially from those prevailing when trials in this review were done.

The operational definition of acute geriatric unit also allowed for variation in the case mix between units, resulting from the existence of other specialised units, mainly intensive care units and coronary units, which attend to patients who would otherwise be cared for in acute geriatric units or transferred to other hospitals. In fact, in three of the randomised trials in this review, the hospitals had specialised units distinct from acute geriatric units ${ }^{\mathrm{w} 3 \mathrm{w} 5 \mathrm{w} 6}$; also certain variation between studies was seen in the diagnostic groups at admission (table). The influence of the case mix on the 


\section{WHAT IS ALREADY KNOWN ON THIS TOPIC}

The effect of geriatric assessment has been evaluated in hospital and community settings

In older people admitted to hospital with acute disorders, the intervention of consultation teams has not shown clinical or administrative benefits

\section{WHAT THIS STUDY ADDS}

Care of older people with acute disorders in acute geriatric units reduces the risk of functional decline at discharge and increases the probability of returning home

This benefit is not accompanied by an increase in case fatality, readmissions, or hospital costs

effectiveness of acute geriatric units should be a priority for future research.

The randomised studies in our review selected patients based on their age (generally $\geq 70$ years). It has been suggested that those patients who will benefit most from acute geriatric units are the frailest elderly, regardless of the condition leading to admission. ${ }^{23}$ Our analysis did not allow us to draw such conclusions, although in most studies evaluated the mean age of the intervention and control groups exceeded 80 years. Four studies reported baseline dependency in at least one basic activity of daily living, which ranged between 40\% and 60\%. ${ }^{\text {w3 w5 w6 w10 }}$ Advanced age and dependence in basic activities of daily living are independently associated with a higher risk of adverse events during hospital stay and of greater functional decline at discharge. ${ }^{192425}$ However, only two studies evaluated the incidence of functional decline in people older and younger than 80 years, and in those who were independent or with some dependence in basic activities of daily living. In both studies the benefit of the intervention was observed in all patient subgroups. ${ }^{\mathrm{w} 3 \mathrm{w} 10}$

\section{Limitations of the study}

This review has some limitations. The number of randomised trials included is small, and the findings of this study might reflect usual clinical practice until 2000 (date of the most recent randomised study in this review). Thus the effect of acute geriatric units compared with conventional hospital units may have changed, as occurred when the results of the first clinical trials of geriatric evaluation and management units were compared with later ones. ${ }^{\text {w38 }}$ However, more recent controlled studies ${ }^{\mathrm{w} 9 \mathrm{w} 10 \mathrm{w} 12}$ suggest that the differences between acute geriatric units and conventional units may still remain. It could be argued that the lack of randomised controlled trials since 2000 is due to acute geriatric units having become the norm for care, so that there are presently no standard hospital care units with which they can be compared. We do not believe this is the case, however, because acute geriatric units are far from widespread in many settings and countries, ${ }^{1326-29}$ and recent research agendas have explicitly recommended to test the effectiveness of this type of care. ${ }^{30}$ In our view the theoretical and practical difficulties of doing these trials might justify the paucity of research in this field. Finally, the studies evaluated do not provide data on the components of the intervention responsible for the benefits observed, nor do they permit us to draw firm conclusions on the effect of acute geriatric units on other relevant outcomes in the medium and long term, except for returning home to live.

\section{Conclusion}

In conclusion, acute geriatric units reduce functional decline at discharge and increase the probability of living at home at discharge and at three months after discharge without increasing case fatality or the costs of hospital care. Research should focus on the impact of acute geriatric units on functional decline in the medium term and should try to identify the specific activities associated with this effect. The methodological quality of investigations should also be improved by giving priority to randomised studies, and efficiency should be evaluated not just by carrying out cost analysis but also by calculating cost effectiveness ratios. Finally, larger sample sizes may be needed to evaluate the impact of acute geriatric units on case fatality.

We thank Mercedes Corrales for her help with the literature search. Contributors: JIB and FRA drafted the manuscript. JJB, FMS, and JLA contributed to the selection of studies and data extraction. All authors contributed to the study design, data analysis, and interpretation of results, and reviewed the manuscript for important intellectual content and approved the final version. JJB is a guarantor.

Funding: This study was partially funded by grant No FIS PI05/90212 and research on fragility and the elderly (RETICEF) grant No RD06/0013, Instituto de Salud Carlos III, Ministry of Health and Consumer Affairs. Competing interests: None declared.

Ethical approval: Not required.

1 Calkins E, Naughton BJ. Care of older people in the hospital. In: Calkins E, Boult C, Wagner EH, Pacala JT, eds. New ways to care for older people. Building systems based on evidence. New York: Springer, 1998:99-111.

2 Ellis G, Langhorne P. Comprehensive geriatric assessment for older hospital patients. Br Med Bull 2005;71:45-9.

3 Parker SG, Fadayevatan R, Lee SD. Acute hospital care for frail older people. Age Ageing 2006;35:551-2.

4 Palmer RM, Counsell SR, Landefeld SC. Acute care for elders units. Practical considerations for optimizing health outcomes. Dis Manage Health Outcomes 2003;11:507-17.

5 Gray L. Geriatric consultation: is there a future? Age Ageing 2007;36:1-2.

6 Shepperd S, Iliffe S. Hospital at home versus in-patient hospital care. Cochrane Database Syst Rev 2005;(3):CD000356.

7 Stuck AE, Siu AL, Wielad GD, Adams J, Rubenstein L. Comprehensive geriatric assessment: a meta-analysis of controlled trials. Lancet 1993;342:1032-6.

8 Parker G, Bhakta P, Katbamna S, Lovett C, Paisley S, Parker S, et al. Best place of care for older people after acute and during subacute illness: a systematic review. J Health Serv Res Policy 2000;5:176-89.

9 Day P, Rasmussen P. What is the evidence for the effectiveness of specialist geriatric services in acute, post-acute and sub-acute settings? A critical appraisal of the literature. NZHTA Report 2004;7(3).

10 Shepperd S, Parkes J, McClaren J, Philips C. Discharge planning from hospital to home. Cochrane Database Syst Rev 2004;(1):CD000313.

11 De Morton NA, Keating JL, Jeffs K. Exercise for acutely hospitalised older medical patients. Cochrane Database Syst Rev 2007;(1):CD005955.

12 González-Montalvo Jl, Baztán Cortés JJ, Alarcón Alarcón T, Bárcena Alvarez A. Rentabilidad de las unidades geriátricas de agudos. Revisión sistemática. Rev Esp Geriatr Gerontol 2007;42:240-50.

13 Jayadevappa R, Bloom BS, Raziano DB, Lavizzo-Mourey R. Dissemination and characteristics of acute care for elders (ACE) units in the United States. Int J Technol Assess Health Care 2003;19:220-7.

14 Sager MA, Franke T, Inouye SK, Landefeld S, Morgan TM, Rudberg MA et al. Functional outcomes of acute medical illness and hospitalization in older persons. Arch Intern Med 1996;156:645-52. 
15 Jadad A, Moore RA, Carroll D, Jenkinson C, Reynolds JM, Gavaghan DJ, et al. Assessing the quality of reports on randomized clinical trials: is blinding necessary? Control Clin Trials 1996;17:1-12.

16 Van Haastregt JCM, Diederiks JPM, van Rossum E, de Witte LP, Crebolder HFJM. Effects of preventive home visits to elderly people living in the community: systematic review. BMJ 2000;320:754-8.

17 Higgins JP, Thompson SG, Deeks JJ, Altman DG. Measuring inconsistency in meta-analyses. BMJ 2003;327:557-60.

18 Altman DG. Confidence intervals. In: Sackett DL, Straus SE, Richardson WS, Rosenberg W, Haynes RB, eds. Evidence-based medicine, 2nd ed. Toronto: Harcourt, 2000:211-20.

19 Reuben DB. Making hospitals better places for sick older persons. Am Geriatr Soc 200;48:1728-9.

20 Salkeld G, Cameron ID, Cumming RG, Easter S, Seymour J, Kurrle SE, et al. Quality of life related to fear of falling and hip fracture in older women: a time trade off study. BMJ 2000;320:241-6.

21 Stroke Unit Trialist' Collaboration. Collaborative systematic review of the randomised of organised inpatients (stroke unit) care after stroke. BMJ 1997;314:1151-9.

22 Cameron I, Crotty M, Currie C, Finnegan T, Gillespie L, Handoll H, et al. Geriatric rehabilitation following fractures in older people: a systematic review. Health Technol Assess 2000;4(2):1-83.

23 Grimley Evans J. Geriatric medicine: a brief history. $B M$ 1997;315:556-61.
24 Covinsky KE, Palmer RM, Fortinsky RH, Counsell SR, Stewart AL, Kresevic D, et al. Loss of independence in activities of daily living in older adults hospitalized with medical illnesses: increased vulnerability with age. J Am Geriatr Soc 2003;51:451-8.

25 Covinsky KE, Justice AC, Rosenthal GE, Palmer RM, Landefeld CS. Measuring prognosis and case mix in hospitalized elders. The importance of functional status. J Gen Intern Med 1997;12:203-8.

26 Bernabei R, Landi F, Zuccala G. Health care for older persons in Italy. Aging Clin Exp Res 2002;14:247-51.

27 Gómez-Pavón J, Ruipérez-Cantera I, Rodríguez-Valcarce A, Rodríguez -Solis J, González-Guerrero JL, Maturana Navarrete N. Level of appropriateness of geriatric resources in general hospitals in Spain: period 2003 to 2005. Rev Esp Geriatr Gerontol 2006;41:77-80.

28 Rockwood K. Commentary: what does an ACE unit trump. Can J Geriatr 2006;9:102.

29 Marin PP. Health policies for older adults: thoughts for action. Rev Med Chil 2007;135:392-8.

30 Norris SL, High K, Gill TM, Hennessy S, Kutner JS, Reuben DR, et al. Health care for older Americans with multiple chronic conditions: a research agenda. J Am Geriatr Soc 2008;56:149-59.

Accepted: 22 October 2008 